\title{
Anisotropy of the Strength Properties of Clays of the North of Belarus
}

\author{
Aliaksander Kremniou$^{1}$, Nikolai Vishniakov ${ }^{2}$ \\ Polotsk State University, Belarus \\ E-mails: ${ }^{1}$ kremnev_si@mail.ru; ${ }^{2}$ n.vishniakov@psu.by (corresponding author)
}

\begin{abstract}
This paper describes the results of experimental investigations anisotropic clay at triaxial tests and uniaxial compression. A band clay of glacial origin has been selected for the study widespread in the north of Belarus. Clays have layered texture due to the conditions of formation. Tests were carried out at different angles to the plane of soil stratification. Test results show significant differences in the values of strength characteristics of soils.
\end{abstract}

Keywords: Soil, anisotropy, strength, clay, triaxial, uniaxial, test.

Conference topic: Soil and rock investigation.

Improving methods of calculation of soil mechanics problems cannot be imagined without improving the methods for the determination of basic mechanical properties of soils. Even the most advanced software systems for the calculation of the foundations can give results that are not conformed with the actual data field tests and observations. The discrepancy between the test data and calculations can sometimes be as high as ten percent or more.

The accuracy of the calculation depends on the input data and the calculation model adopted by the soil. The main sources of data for the calculation of the foundations are the mechanical properties of soils. These are very important features of sedimentary soils which are not always taken into account while determining them particularly this anisotropy of mechanical properties. The same applies to the calculation model. In classical soil mechanics the soil is considered to be a continuous isotropic medium which properties do not change with change of direction of the principal stresses.

Many studies show that most of the soils have the anisotropy. This is especially true for the soils with an obvious stratification. Now we can say that soils have the possess of anisotropy with pronounced stratification, but also without it. Soil anisotropy studied today by many scientists. Here are some of them (Roosjen et al. 2015; Zhao, Guo 2015; Wang et al. 2015).

We can select different types of anisotropy. They are the following deformation, seepage, anisotropy of the strength properties and the anisotropy of specific characteristics: swelling, heat conduction, wave propagation velocity, etc.

This article presents the results of investigations of the anisotropy of the strength properties of clays of glacial origin which are widespread in the North of Belarus, in the conditions of triaxial and uniaxial tests. We investigated anisotropy of sandy soils in our previous work (Kremniou, Vishniakov 2008). The studied soils had a pronounced anisotropy due to the conditions of their formation.
These formations belong to the period of Lace glacier. They were formed in glacial lakes, which are the batteries of the fine-clastic material (Pavlovskaya 1994).

Firstly, the anisotropy of these soils is due to the layered texture of soil associated with the seasonal inflow of detritus and secondly, the predominant orientation of the soil particles which have a large axis parallel to the horizon as studies show (Pavlovskaya 1994).

Thus, tape clays have a mostly plane (direction) stratification caused by the formation of ground conditions. So the soil can be regarded as a transversely isotropic medium where the strength characteristics of ground will vary depending on the angle of inclination of the plane of stratification to the shear plane. Therefore, the determination of the strength characteristics of soils is required in at least two directions: along and across stratification.

Triaxial tests and uniaxial compression tests were conducted to confirm the presence of anisotropy of the strength properties of the studied soils. Determination of the strength characteristics of the clay was carried out in the triaxial apparatus in two mutually perpendicular directions. Specimens were taken in two mutually perpendicular directions, ie, along and across stratification plane. The size of the specimens was $38 \mathrm{~mm}$ - diameter $76 \mathrm{~mm}$ - height.

Triaxial tests were carried out by consolidateddrained scheme for determining the strength characteristics.

Mohr-Coulomb diagrams were constructed to determine the strength properties of soils after statistical processing of the results (Fig. 1).

Strength properties were obtained as follows:

- for specimens with horizontal stratification $\varphi=3^{0}, \mathrm{c}=18 \mathrm{kPa}$;

- for specimens with vertical stratification $\varphi=7^{0}$, $\mathrm{c}=22 \mathrm{kPa}$. 
a)

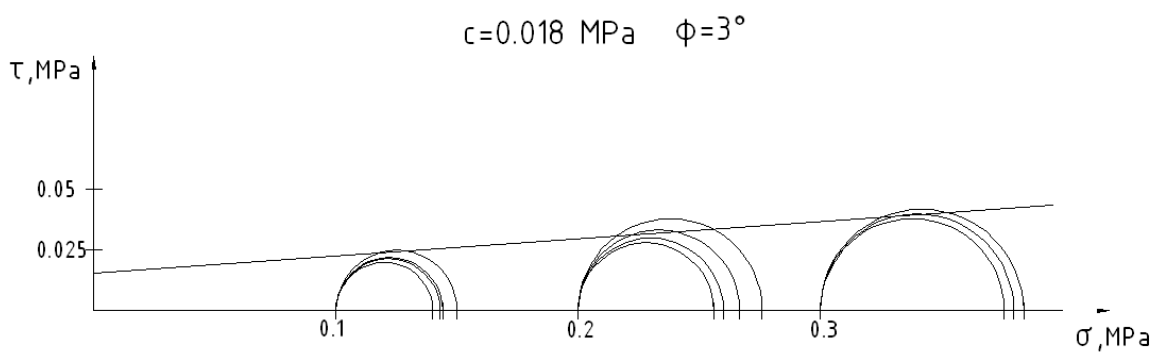

b)

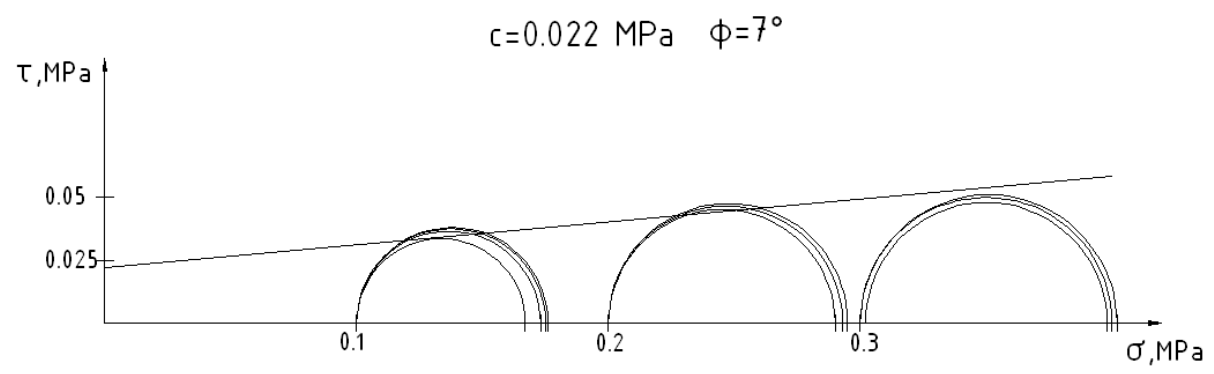

a) specimens with horizontal layering, b) specimens with vertical layering

Fig. 1. Diagrams the Mohr-Coulomb

Breaking vertical load was recorded during the test in the uniaxial compression. Instant shift one part of the sample with respect to the other, or the achievement of the overall relative sample deformation 0.15 was taken as the destruction of the samples. The test results are shown in Figure 2 as a function of relative vertical deformation of vertical stress. Photos of the samples tested are shown in Figure 3.

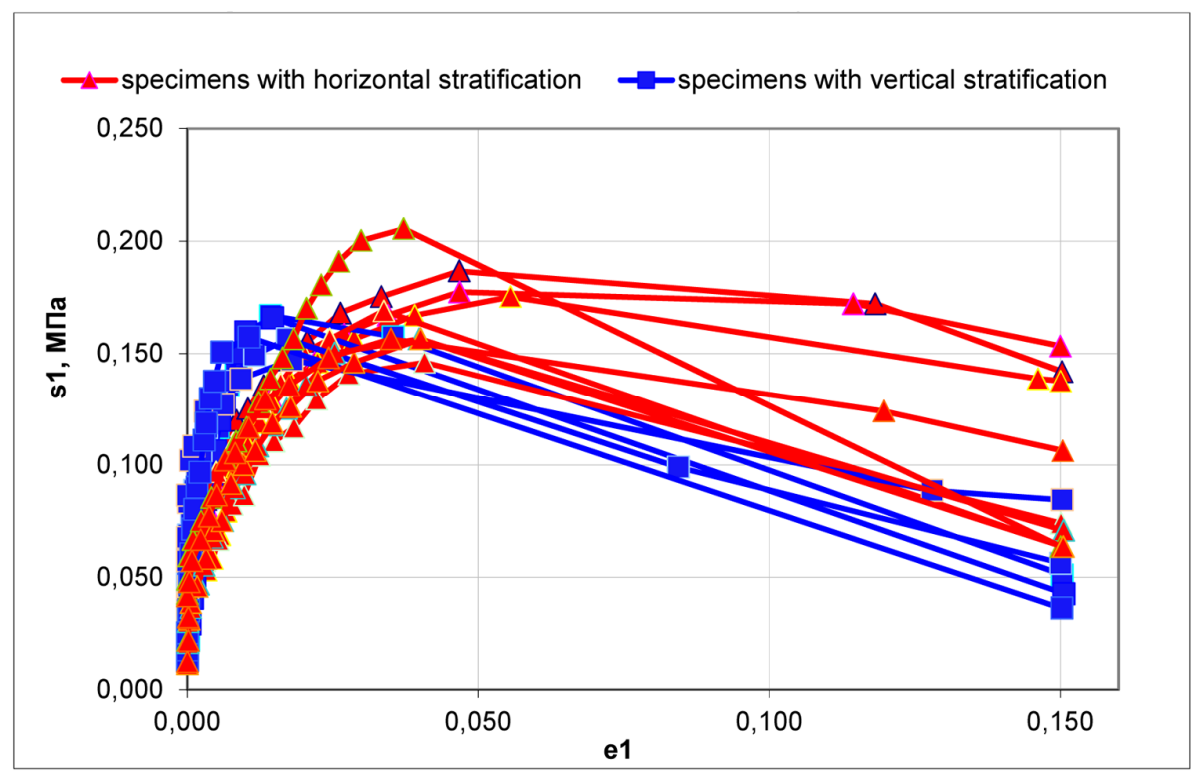

Fig. 2. The results of the soil tests on uniaxial compression 


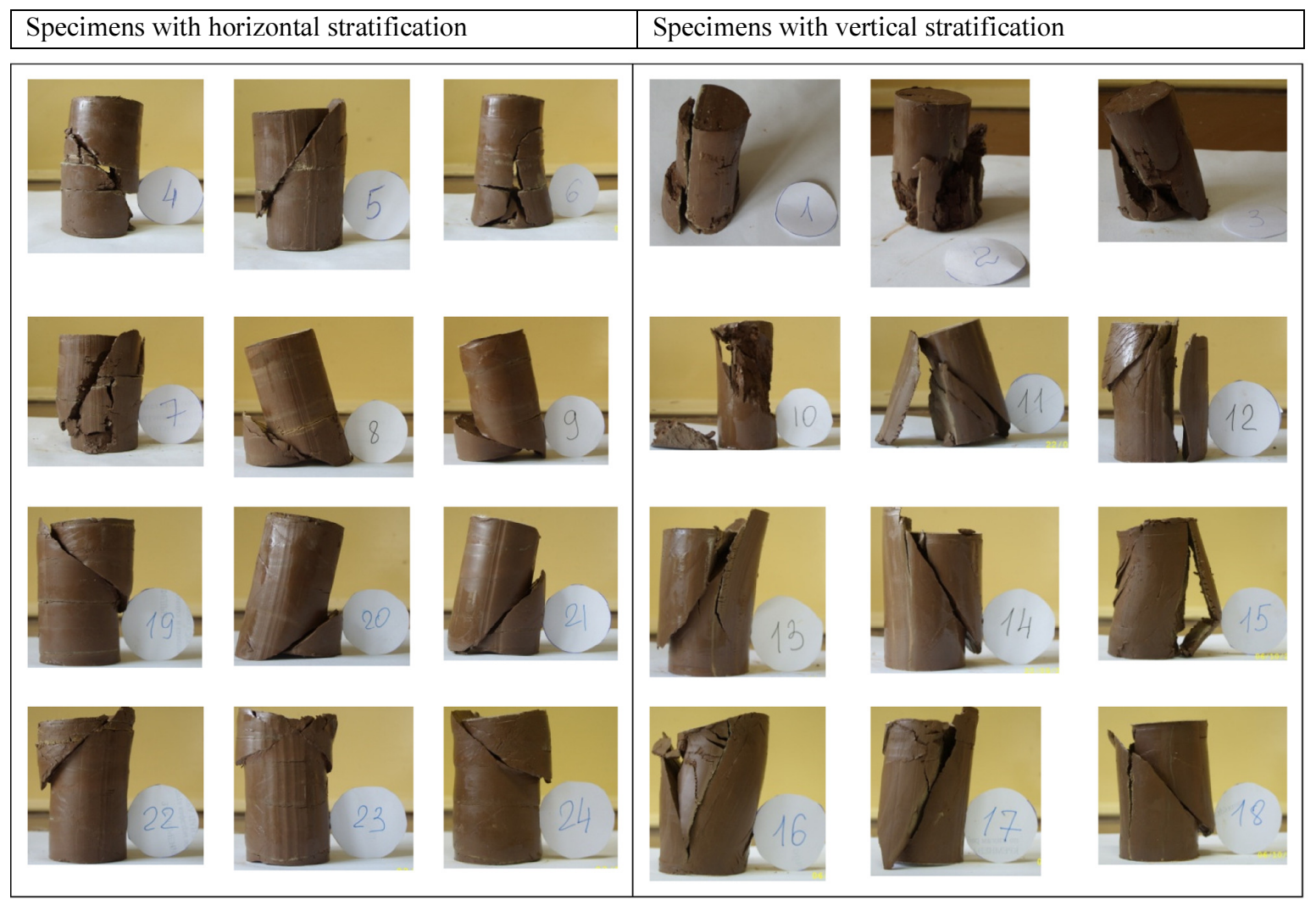

Fig. 3. Specimens after the test uniaxial compression

Derived value of tensile strength were obtained by the test results:

- for specimens with horizontal stratification $0.1579 \mathrm{MPa}$;

- for specimens with vertical stratification $0.1407 \mathrm{MPa}$.

After analyzing the derived value on uniaxial compression it can be concluded that the test sample with a horizontal arrangement of stratification has the strength at about $11 \%$ more than at the location of stratification perpendicular to the horizon.

Influence of anisotropy of the strength properties of the soil is the most significant impact on the accuracy of determining limit loads on the basis of the foundations of the spacer structures experiencing significant horizontal loads. Examples of constructions, foundations which are working on the horizontal loads are amphitheater, arched structures and waterworks. Determination of limit load on the base without taking into account the anisotropy of the strength properties can lead to significant errors in this case.

Sliding surfaces cross stratification axis at different angles in solving the Prandtl and method of slices (limit equilibrium method). Therefore it is necessary to know the pattern of change of the angle of internal friction and specific cohesion values depending on the orientation of the shear surface with respect to the stratification axis.

Equation Casagrande-Carrillo was one of the first to determine the dependency of anisotropic properties of soil by direct shear:

$$
\mathrm{S}_{\alpha}=\mathrm{S}_{0}+\left(\mathrm{S}_{90}-\mathrm{S}_{0}\right) \sin ^{2} \propto,
$$

where $\alpha$-the angle of the shear plane to the plane of the stratification; $S_{0}, S_{90}$ - strength (shear strength) on the cut planes with angles $\alpha=0^{\circ}$ and $\alpha=90^{\circ}$, i.e., along and across to the stratification axis.

Ivanov gives a similar dependence for determining the strength characteristics of the soil (Ivanov 1991):

$$
\begin{gathered}
\operatorname{tg} \varphi_{\alpha}=\operatorname{tg} \varphi_{1}+\left(\operatorname{tg} \varphi_{2}-\operatorname{tg} \varphi_{1}\right) \sin ^{2} \alpha \\
c_{\alpha}=c_{1}+\left(c_{2}-c_{1}\right) \sin ^{2} \alpha
\end{gathered}
$$

where $\alpha$-the angle of the shear plane to the plane of the stratification; $\operatorname{tg} \varphi_{1}, c_{1}$ - shear strength characteristics in the direction of stratification (along the plane of isotropy); $\operatorname{tg} \varphi_{2}, c_{2}-$ the same shear across the stratification (across the plane of isotropy).

According to Bugrov and Golubev (1993), the Eqs (1) and (2) are widely used in the calculations and 
the accuracy of this dependence was analyzed in many studies.

The critical load on the foundation can be determined by the method of slices (limit equilibrium method), knowing the character of the effect of anisotropy on the mechanical properties of the soil. The values of specific cohesion and angle of internal friction of soil are taken in view of the proposed angle of inclination of the sliding surface and the direction of stratification. The task of determining slope stability is achieved in a similar manner.

\section{Conclusions}

- Clay glacial origin has a pronounced stratification caused by the conditions of their formation.

- It was established experimentally that the strength properties of the glacial lake clays depend on the orientation of the direction of principal stresses with respect to the axis of the soil stratification.

- At triaxial tests the greatest value of the internal angle of friction and specific cohesion were achieved by stratification the location across the action of principal stress $\sigma 1$.

- By uniaxial compression in the test sample with a horizontal arrangement of stratification its strength was about $11 \%$ more than at the location of stratification perpendicular to the horizon.

- The effect of anisotropy on strength properties of the soil must be considered when determining the critical load on the base and especially for spacer structures foundations experiencing substantial horizontal loads. In this case the value of the limit load may be obtained by method the of slices (limit equilibrium method) surfaces.

\section{References}

Bugrov, A.; Golubev, A. 1993. Anisotropic soils and foundation structures. St. Petersburg: Nedra.

Ivanov, P. 1991. Soils and grounds waterworks. Moscow.

Kremniou, A.; Vishniakov, N. 2008. The anisotropy of the strength properties of sandy soils, in Collected papers of International Scientific Conference "Geotechnics Belarus: Science and Practice", 20-22 October 2008, Minsk, Belarus. BNTU, 19-26.

Pavlovskaya, I. 1994. Polotsk glacial lake basin: the structure, topography, history of development. Minsk: Science and Technology.

Roosjen, P. P. J.; Bartholomeus, H. M.; Clevers, J. G. P. W. 2015. Effects of soil moisture content on reflectance anisotropy - Laboratory goniometer measurements and RPV model inversions, Remote Sensing of Environment 170: 229-238.

http://dx.doi.org/10.1016/j.rse.2015.09.022

Wang, B.; Xiao, H.; Dang, Y.; Sun, L. 2015. Influence of the anisotropy of a transversely isotropic foundation on additional stress fields, Sichuan Daxue Xuebao (Gongcheng Kexue Ban)/Journal of Sichuan University (Engineering Science Edition) 47(6): 40-48.

Zhao, J.; Guo, N. 2015. The interplay between anisotropy and strain localisation in granular soils: a multiscale insight, Geotechnique 65(8): 642-656.

http://dx.doi.org/10.1680/geot.14.P.184 